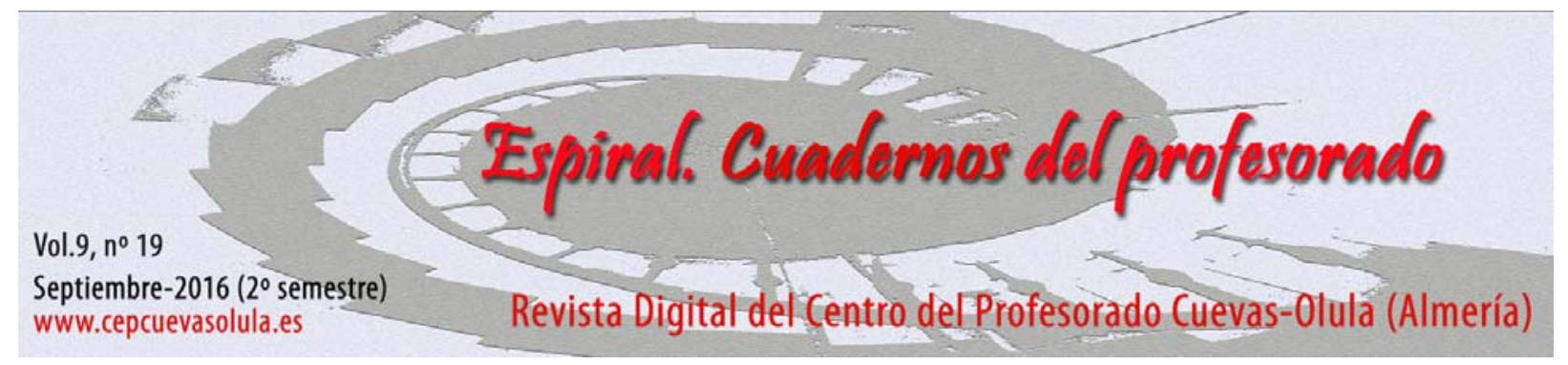

\title{
PERCEPCIONES DE PROFESORADO DE SECUNDARIA SOBRE LAS PUBLICACIONES DE DIDÁCTICA. EXPERIENCIAS EN LA FORMACIÓN CONTINUA
}

\author{
Perceptions of Secondary Education teachers about pedagogy publications. Experiences \\ in in-service teacher training
}

\section{Jordi Domènech Casal}

Instituto de Secundaria de Granollers, Barcelona, España

Departamento de Didáctica de las Matemáticas y las Ciencias Experimentales, Universitat Autònoma de Barcelona, España

\begin{abstract}
RESUMEN: Se describe una experiencia de inclusión de revistas nacionales de didáctica en cuatro actividades de formación del profesorado. Los resultados recogidos en una encuesta se discuten junto con el análisis de una breve experiencia de difusión de publicaciones de didáctica mediante las TIC y un análisis del contenido de varias revistas de didáctica específica de las ciencias. Los resultados indican que el profesorado, a pesar de no usarlos a menudo, valora positivamente los artículos de didáctica como recurso, y prefiere las revistas técnico-profesionales a las académicas, y usa tanto los artículos de descripción de actividades como los de descripción de marcos pedagógicos teóricos. La discusión en grupo en torno a un proyecto compartido y la libre elección de artículos promueven una mejor relación profesional con las publicaciones de didáctica. Se ofrecen pautas e indicaciones para el uso de publicaciones de didáctica en la formación continua para conectar la teoría con la práctica.
\end{abstract}

Palabras clave: formación continua, gestión del conocimiento, desarrollo profesional, investigación-acción, revistas de didáctica.

\begin{abstract}
The teaching guide is a basic tool to reach the Convergence process guiding principles in the European Space of Higher Education (EEES). Each subject design should follow a double way: the content and the student work related to this content. Therefore, a teaching guide should specify the academic content selection and ordination, how will these contents be worked out, and the students evaluation method. Our teaching guide is an approach about the competences students should acquire, the subject content structure, what to teach and learn from these contents, the educational development to reach the learning, the student dedication and the learning evaluation and score methods, in the subject Data Analysis from the Degree in Sciences of Physical Activity and Sports of the San Antonio Catholic University of Murcia (UCAM), to advantage the students teaching-learning process.
\end{abstract}

Key words: continuous training, knowledge management, profesional development, action-research, Pedagogy journals.

Domènech Casal, J. (2016). Percepciones de profesorado de secundaria sobre las publicaciones de didáctica. Experiencias en la formación continua. Espiral. Cuadernos del Profesorado, 9(19), 3-18. Disponible en: http://espiral.cepcuevasolula.es/

Fecha de recepción: 01/08/2016

Fecha de aceptación: 18/09/2016
Enviar correspondencia a: jdomen44@xtec.cat 


\section{Introducción}

Los modelos tradicionales de formación continua del profesorado, fundamentados en estrategias transmisivas y roles de expertos/aprendices, hacen compleja la aplicación en las aulas de los ejes formativos (Perrenoud, 2010). En estos modelos, que podríamos llamar formación $e$ innovación 1.0 (Domènech-Casal, 2016), el conocimiento pedagógico es transmitido de forma vertical desde la administración o las instituciones académicas y el profesorado participa como receptor y aplicador de propuestas que no contribuye a construir. Estos modelos verticales de formación e innovación, bastantes habituales en la formación del profesorado (Furió y Gil, 1998), suelen comportar un alejamiento de los actuantes (profesorado) y las fuentes del conocimiento.

Por ello, varios autores expresan la necesidad de que la formación e innovación implique un rol más activo por parte del profesorado.y más conectado a la experiencia propia, $\mathrm{y}$, en particular, a la reflexión sobre su propia acción (Zeichner, 1993; Guisasola, Pintos y Santos, 2001; Perrenoud, 2010) de forma conectada a su centro escolar y sus prácticas previas (OECD, 2015, Barba-Martín, Barba y Martínez, 2016), si bien se considera también puede desarrollarse entre centros cercanos, en lo que Heargreaves y Shirley (2012) denominan transferencia lateral, que suele producirse en grupos de trabajo de ámbito disciplinar formados por docentes de distintos centros.

Eso implica una visión horizontal de la formación e innovación en la que son los propios actuantes los que construyen el conocimiento profesional en lo que podemos llamar formación e innovación 2.0 (Domènech-Casal, 2016). Este modelo implica cambios en la gestión del conocimiento y estructuras organizativas del centro educativo (Lavié, 2012; Rodríguez-Gómez, 2011), pero también en el diseño de la formación del profesorado, que debe orientarse más a la cooperación, la simetría, la meritocracia, la descentralización y la multilateralidad (Imbernón, 2002). Entre estas propuestas encontramos las conocidas como investigación-acción y formación entre iguales e implican el desarrollo de un rol profesional y de competencias profesionales específicas en investigación e innovación educativa (Andreis, 2013; Perrenoud, 2010; Tejada, 2009) y suelen materializarse en forma de grupos de trabajo o encuentros o jornadas de intercambio de experiencias que emulan los formatos de los congresos científicos. En estos espacios, la formación deja de ser un evento profesional conducido "desde arriba" y "desde fuera" a ser conectado "desde dentro" y "desde abajo" por un profesorado que lidera su formación al tiempo que se capacita en innovación (Palos, Martínez y Albadalejo, 2012; Tejada 2009). También en el ámbito institucional impactan la reacciones a este cambio: los ejes para el desarrollo de un rol profesional, como la reciente propuesta del "Libro Blanco de la profesión Docente" (Marina, Pellicer y Manso, 2015) se articulan, precisamente, en una formación continua, autónoma y vinculada a la observación de la propia práctica.

Una de las dificultades que implica este salto, en ocasiones realizado a ciegas y sin aprovechar el conocimiento epistemológico previo de las experiencias de investigación-acción y formación entre iguales, es su falta de conexión con referentes pedagógicos y discursos excesivamente centrados en lo técnico (o tecnológico), como ha ocurrido en los últimos años con la formación de redes y proyectos de innovación TIC centrados en el aparataje y vacíos de contenido pedagógico o social (Sancho y Alonso, 2012). De hecho, otros autores describen que el profesorado mantiene sus prácticas impermeables a los resultados de la investigación educativa (Solbes et al, 2013).

Por esta razón existe una necesidad de conectar las iniciativas de formación entre iguales al resto de actores del panorama educativo (universidad, fundaciones,...) y referentes pedagógicos, una necesidad especialmente acuciante en el profesorado de secundaria, que tradicionalmente ha accedido a la profesión con poca o muy poca formación en pedagogía y didáctica (Carrascosa-Alís, MartínezTorregrossa, Furió-Mas y Guisasola, 2008; European Comission, 2015) y sin los referentes conceptuales (líneas pedagógicas y discursos sociales en educación), procedimentales (cómo analizar fenómenos didácticos, qué fuentes bibliográficas existen) ni epistemológicos (cómo se construye y de qué naturaleza es el conocimiento pedagógico). Unas carencias formativas que generan en el profesorado falta de confianza en su inmersión en dinámicas de innovación e investigación (Oliva, 2011). 


\section{Escribir y leer textos de didáctica desde la práctica}

Las revistas de didáctica general y específica suponen una oportunidad de establecer una conexión actualizada con estos tres tipos de referentes en la formación inicial y continua del profesorado. Esto incluye dos tipos de revistas: las revistas académicas (que suelen publicarse en abierto desde instituciones universitarias en formato científico y en las que escriben, mayoritariamente, académicos) y las revistas técnico-profesionales (a menudo publicadas por editoriales o entes vinculados a la administración, en cerrado, y en las que escriben maestros, profesores de secundaria y académicos en un estilo más divulgativo).

Está descrito en la literatura que la participación del profesorado en la escritura de textos de reflexión sobre la práctica es una herramienta muy útil para la reflexión, en forma de diarios del docente, autoetnografías (Barba, González-Calvo y Barba-Martín, 2014, Moon, 2006), o incluso artículos de didáctica resultantes de la propia práctica, lo que redunda en el desarrollo de un rol profesional y establecer una relación más cordial de los conocimientos "técnicos" con los "académicos" (Andreis, 2013). Esto facilita también en que las innovaciones puedan difundirse y ser de utilidad para otras instituciones educativas (Greenwood, 2000). Aún así, esta valoración positiva no es compartida de manera global por el profesorado: en una encuesta reciente, sólo un $10 \%$ del profesorado valora en una innovación su publicación (Sein-Echaluce, García y Fidalgo, 2014). Así mismo, los profesores encontramos dificultades para participar en la escritura y comunicación de innovaciones principalmente por la falta de fundamentación teórica, y aspectos prácticos de la redacción escrita del trabajo realizado (Oliva, 2012).

En lo que respecta a la lectura o consulta de publicaciones de didáctica, si bien en la formación inicial el profesorado tiene contacto con las publicaciones de didáctica, su uso está mucho menos extendido en la formación continua, llegando a la situación normalizada en la que se hacen cursos de formación a profesores -todos ellos con estudios universitarios- sin ningún tipo de bibliografía ni información sobre investigación educativa (Carro, 2000). Incluso en experiencias de investigaciónacción y práctica reflexiva, son frecuentes los casos en los que no se menciona en ningún momento como parte de la dinámica la consulta de artículos de didáctica como fuente de discusión para crear conocimiento, pues éste parece "emanar" o "construirse" exclusivamente desde la práctica y la interacción entre participantes. De modo que el profesorado de secundaria se suele encontrar inmerso en actividades formativas "suaves" de propuestas pedagógicas institucionales (como la de las Competencias Básicas o la TIC) sin tener acceso formativo a las bases pedagógicas que las engendraron (construccionismo, conectivismo...) ni las vías de continuidad o profesionalización que se reclaman desde las propias instituciones educativas (OECD, 2013). Como consecuencia, el profesorado no recurre a las revistas de didáctica como fuente, como muestra una encuesta reciente a más de 2200 profesores, en la que se detecta que el profesorado obtiene información útil primeramente en web 2.0 y cursos de formación, en segundo lugar en congresos y seminarios, y sólo en tercer lugar en revistas científicas (Sein-Echaluce, García y Fidalgo, 2014).

La ausencia de las publicaciones de didáctica o pedagogía en los cursos de formación contribuye a agravar la concepción habitual según la cual las publicaciones de didáctica o pedagogía (o, incluso, la Pedagogía en sí) están alejadas de la práctica y no resuelven los problemas reales en el aula (Imbernón, 2002; Sanmartí, 2008). Esta percepción puede deberse a dos razones: por un lado, las razones descritas por otros autores (Sanmartí, 2008), en el sentido que el profesorado tiene dificultad para entender los artículos porque comparte pocos referentes teóricos y no conoce el género lingüístico. Paradójicamente la especialización de las revistas para mejorar en el ranquin hace que éstas dejen de ser leídas por el profesorado (Sanmartí, 2008). A ello debe añadirse las dificultades de acceso que experimentan los docentes en revistas que no son publicadas en abierto. Por otro lado, la labor de búsqueda y selección de artículos en distintas revistas puede resultar descorazonadora para un profesor que, por ejemplo, desea mejorar su enseñanza de una temática concreta. Como muestra un estudio reciente (INEE, 2014), el profesorado dedica sólo un 6,8\% de su tiempo a planificación (y el estudio ni tan sólo incluye una categoría de "investigación" o "actualización"), por lo que esta falta de 
espacios y tiempos de reflexión hace que sea poco realista esperar que el profesorado en general dedique su tiempo a "leer para formarse" de forma genérica ante la urgencia del día a día.

En conjunto, el uso de las revistas de didáctica como espacio de emancipación y contacto directo con las fuentes académicas presenta dos problemas principales: 1) La falta de espacios de discusión entre iguales a partir de artículos de didáctica -y no sólo de la propia práctica- y 2) El exceso de especialización de las revistas, que no son ya abordables para el profesorado, por su contenido excesivamente especializado o teórico.

\section{Antecedentes y objetivos}

Dada la importancia del trabajo en equipo del profesorado y la lectura de revistas de didáctica y pedagogía, se optó por llevar a cabo unos Seminarios de Lectura de artículos de revistas de didáctica. Éstos se llevaron a cabo en un grupo de trabajo de profesorado (EduWikiLab ${ }^{1}$ ) formado los cursos 2013-2014 y 2014-2015 por profesores de ciencias de 10 centros educativos de la provincia de Barcelona. Durante el curso 2013-2014, la actividad se llevó a cabo de forma tentativa. El curso 20142015 se consolidó y se colectaron evidencias para el análisis, ampliando la experiencia bajo otros formatos en otras actividades formativas como se describe en la metodología. En paralelo se analizaron los contenidos de varias revistas de didáctica específica de las ciencias y el comportamiento de los usuarios de nuevas tecnologías ante contenidos digitales con artículos de revista de didáctica. El conjunto de la experiencia tiene como objetivos:

- Explorar vías de conexión de la formación continua del profesorado con las publicaciones de didáctica y pedagogía.

- Identificar variables en el uso de publicaciones de didáctica y pedagogía (espacio de discusión, acceso, posibilidad de elección, utilidad de los contenidos).

- Evaluar las percepciones del profesorado de secundaria sobre las publicaciones de didáctica y pedagogía.

\section{Metodología}

\section{Diseño y muestra}

Valoración de las publicaciones de didáctica por parte del profesorado

Se llevaron a cabo 4 experiencias de inclusión de revistas de didáctica en distintas modalidades. En cada modalidad los artículos fueron incluidos con una dinámica distinta en función de la presencia/ausencia de un espacio de discusión colectiva y la posibilidad de elección), que describimos a continuación:

Modalidad Discusión en marco formativo: (Espacio colectivo de discusión, elección libre). En el marco del grupo de trabajo de profesorado de ciencias EduWikiLab, se llevaron a cabo los Seminarios de Lectura de Didáctica ${ }^{2}$, cinco encuentros de discusión de artículos de didáctica de las ciencias repartidos a lo largo del curso 2014-2015 en los que al iniciar cada sesión cada uno de los 14 miembros del grupo de trabajo comentaba brevemente $(3 \mathrm{~min})$ un artículo de didáctica de elección libre que hubiera resultado de su interés, describiendo su contenido y justificando su interés para la mejora de la enseñanza de las ciencias. Se puso a disposición de los participantes una "librería digital" de revistas de didáctica específica de las Ciencias publicadas en abierto construida ad hoc (muestra de revistas descrita en el siguiente subapartado). Las revistas propuestas lo fueron en base a 3 criterios: publicadas en español, accesibles en abierto de forma digital, y centradas en el ámbito de la formación, la didáctica de las ciencias.

Modalidad Acompañamiento en marco formativo: (Espacio colectivo de discusión, elección limitada).en el marco de dos acciones formativas distintas -Formación Ventanas Moodle en Educación Inclusiva y Altas Capacidades- en el Instituto de Canet de Mar (15 profesores) y Formación Intercentros IndComp sobre evaluación de Competencias Básicas (12 profesores) (Armengou et al, 
2015) - se propuso como tarea de la formación la lectura de artículos específicos de revistas de didáctica general y pedagogía (Cuadernos de Pedagogía, Aula de Secundaria), que debían comentarse en formato digital (foro) o presencial (debate) como parte de la formación. Los participantes debían elegir entre varios artículos propuestos de una lista cerrada, parte de los cuales se consiguieron gracias a la colaboración de la revista Cuadernos de Pedagogía, que ofreció en abierto un conjunto específico de artículos a propósito para su uso en la actividad formativa. Los artículos propuestos fueron seleccionados en base a su pertenencia a revistas de ámbito general, publicados en español y tratando el tema de la programación y evaluación por Competencias.

Modalidad Disponibilidad: (Sin espacio de discusión, elección limitada) en el Instituto de secundaria de Vilanova del Vallès (Barcelona) se pusieron durante el curso 2014-2015 a disposición del profesorado (50 profesores de secundaria de distintas materias) los números del año en curso de 4 revistas de didáctica general y específica (Cuadernos de Pedagogía, Alambique, Science in School, Aula de Secundaria). Las revistas se exponían en un espacio expreso en la Sala de Profesores y se anunciaba mediante correo electrónico general la llegada de un nuevo número, comentando su contenido. Aunque en paralelo se realizaba en el centro una formación sobre TICs, la propuesta de las revistas se hizo de forma desvinculada a la formación específica y se limitó a hacer accesibles las revistas.

Modalidad Invitación en marco formativo: (Sin espacio de discusión, elección libre) en el marco de una formación específica en enseñanza de las ciencias a profesorado en activo organizada por la Fundació Catalana per la Recerca i la Innovació (Experimentació a les Aules ${ }^{1}$ ) se describieron los contenidos y recomendaron a 75 docentes varias revistas de didácticas específicas de las ciencias (Enseñanza de las Ciencias, Enseñanza de las Ciencias de la Tierra, Revista Ciències, Educació Química, Recursos de Física, Revista Eureka de Enseñanza de las Ciencias, Revista Electrónica de Enseñanza de las Ciencias), argumentando su utilidad, pero sin desarrollar ninguna actividad ni acompañamiento. Las revistas propuestas lo fueron en base a 3 criterios: publicadas en español, accesibles en abierto de forma digital, y centradas en el ámbito de la formación, la didáctica de las ciencias.

\section{Análisis de las elecciones de artículos de didáctica por parte de los profesores}

Se creó una "librería digital" con los accesos a distintas revistas de Didáctica de las Ciencias. Se ofreció su acceso a los participantes en el grupo de trabajo EduWikiLab para hacer un seguimiento de sus elecciones. Se incluyeron las siguientes revistas:

$\checkmark$ Revista Eureka sobre Enseñanza y Divulgación de las Ciencias

$\checkmark$ Revista Ciències, Revista del Professorat de Ciències d'Infantil, Primària i Secundària

$\checkmark$ Recursos de Física

$\checkmark$ Science in School

$\checkmark$ Revista Electrónica de Enseñanza de las Ciencias

$\checkmark$ Enseñanza de las Ciencias de la Tierra

$\checkmark$ Enseñanza de las Ciencias

$\checkmark$ Educació Química

$\checkmark$ Didáctica de las Ciencias Experimentales y Sociales

Incorporadas durante la modalidad de Discusión:

$\checkmark$ PLOS ONE. Science Education

$\checkmark$ European Journal of Physics

Las revistas propuestas lo fueron en base a 3 criterios: publicadas en español, accesibles en abierto de forma digital, y centradas en el ámbito de la formación, la didáctica

1 Curso Experimentació a les Aules de Ciències, de la FCRI http://www.recercaenaccio.cat/wpcontent/uploads/2015/05/UB estiu2015.pdf 
de las ciencias. El conjunto de enlaces se puso a disposición del profesorado en la forma de una "Biblioteca virtual" mediante enlaces en el espacio web del grupo de trabajo ${ }^{3}$.

Se colectaron 42 artículos elegidos por los participantes, registrados en el espacio wiki creado ad hoc (http://eduwikilab1415.wikispaces.com/Seminaris+de+lectura) en el que cada participante del grupo de trabajo incorporaba la referencia del artículo de su elección y un breve comentario.

Análisis del contenido de revistas de didáctica específica de las ciencias

Con el objetivo de analizar el contenido de varias publicaciones de didáctica específica, se analizaron 300 artículos publicados en los últimos números de revistas de didáctica de las ciencias. Las revistas propuestas lo fueron en base a 3 criterios: publicadas en español, accesibles en abierto de forma digital, y centradas en el ámbito de la didáctica de las ciencias. Las revistas (y números) analizados se detallan en la Tabla 1. Se leyeron los resúmenes y conclusiones de todos ellos, y se agruparon según las temáticas, tal como se describe en Instrumentos, recogida y análisis de datos.

Tabla 1.

Números revisados según el contenido ofrecido por los artículos.

\begin{tabular}{lc}
\hline \multicolumn{1}{c}{ Revista } & Números analizados \\
\hline Alambique, Didáctica de las Ciencias Experimentales & $79,80,81$ \\
Enseñanza de las Ciencias & $33(1,2,3)$ \\
Revista Electrónica de Enseñanza de las Ciencias & $13(1,2,3), 14(1,2)$ \\
Revista Eureka de Enseñanza y Divulgación de las Ciencias & $11(3), 12(1,2)$ \\
Revista Ciències & $26,27,28,29$ \\
Enseñanza de las Ciencias de la Tierra & $21(1,2,3), 22(1)$ \\
Science in School & $30,31,32$ \\
Recursos de Física & $11,12,13,14,15$ \\
\hline
\end{tabular}

Nota. Se han analizado distinta cantidad de números en cada revista en función del número de artículos por ejemplar, con la intención de revisar una cantidad similar de artículos de cada revista.

Inclusión en entorno TIC y análisis de cibermetría

Se prepararon 4 textos, cada uno con una recopilación de varios (4-5) artículos de revistas de didáctica sobre un tema de interés en didáctica de las ciencias (evaluación, indagación, formulación de preguntas, lectura en ciencias), incluyendo en cada texto 4-5 enlaces a artículos en abierto de revistas de didáctica y un breve resumen-presentación de los contenidos. Los textos se publicaron en marzo de 2015 en forma de post independientes en el blog educativo Pupitrelàndia ${ }^{2}$. De forma paralela, los distintos posts fueron difundidos mediante una cuenta Twitter.

\section{Instrumentos, recogida y análisis de datos}

\section{Valoración de las publicaciones de didáctica por parte del profesorado}

Al terminar cada una de las actividades formativas de las distintas modalidades (Discusión en marco formativo, Acompañamiento en marco formativo, Disponibilidad, Invitación en marco formativo,), se pasó a los participantes la siguiente encuesta:

\section{Cuestionario}

Responder valorando del 1 (completamente en desacuerdo) al 6 (completamente de acuerdo).

2 Post-Recopilatorios de artículos en el blog Pupitrelàndia https://blogcienciesnaturals.wordpress.com/tag/compilacions-tematiques/ 
- Los artículos de didáctica tratan de cosas directamente relacionadas con mi trabajo

- Leer artículos de didáctica es una ayuda para resolver problemas prácticos

- Leer artículos hace que valore más las cosas que hago

- Leer artículos hace que me fije más en las cosas que hago

- Participar en la escritura de artículos ayudaría a analizar las actividades que hago

- Participar en la escritura de artículos puede mejorar la percepción que tengo de mi oficio

- Considero que puedo recurrir a leer artículos como herramienta de trabajo

— Las publicaciones de didáctica deberían estar más presentes en la vida diaria de los centros

- Los artículos de didáctica son suficientemente accesibles para el profesorado

- Los artículos de didáctica deberían formar parte de la formación inicial y continua

— Los artículos de didáctica me permiten formarme según mis intereses y necesidades

- Leer o escribir artículos de didáctica me ayuda a formarme una idea de mi identidad como profesional de la educación.

— En lo referente artículos de didáctica, cada curso académico consulto/leo un total de..........artículo/s.

Se recogieron un total de 83 encuestas de las distintas modalidades al final de la experiencia formativa. Se calculó de forma separada para cada modalidad la media de apoyo que recibía cada frase. La pregunta 13 no se hallaba en los cuestionarios iniciales, por lo que fue respondida por 61 profesores.

\section{Análisis de las elecciones de artículos de didáctica por parte de los profesores}

Se clasificaron los artículos en una de las dos categorías: Propuestas didácticas prácticas /Marcos teóricos o pedagógicos, y de manera transversal, se agruparon según temática específica en 6 ámbitos (TIC y Multimedia, Lectura, escritura y oralidad en ciencias, Comp. Básicas y PISA, Evaluación, Laboratorio y actividades manipulativas, Indagación). Si bien los ámbitos no son exclusivos, se intentó formar ámbitos lo más grandes posibles tomando en consideración los aspectos principales de cada artículo.

\section{Análisis del contenido de revistas de didáctica específica de las ciencias}

Los artículos de las distintas revistas se agruparon según su temática, independientemente de la revista de procedencia, resultando 7 categorías:

- Concepciones y análisis de alumnado de primaria. / secundaria.

- Análisis de libros de texto.

- Currículum.

- Concepciones y análisis de alumnado de magisterio.

- Propuestas didácticas de aula.

- Marcos teóricos o pedagógicos.

- Concepciones y análisis de profesorado de primaria/secundaria.

Se recogieron y agruparon por categorías según su temática los 42 artículos elegidos por el profesorado, junto con los comentarios suscitados.

\section{Inclusión en entorno TIC y análisis de cibermetría}

En agosto de 2015, cinco meses después de su publicación, se midió el número de visitas de cada uno de los posts publicados con las recopilaciones de artículos de didáctica. Se calculó el número 
de visitas medio de otros posts de antigüedad y difusión equivalente. Se midieron también el número de veces que cada uno de los enlaces a un artículo de didáctica incluido en el post era activado por el lector del post.

En paralelo se contabilizó el \% de usuarios que, al leer uno de los Tuits difundiendo una recopilación de artículos de didáctica, realizaban alguna acción (abrir el enlace, ampliar el Tuit, clicar sobre el nombre del usuario (\%Engagement...). Se comparó el \%Engagement obtenido con el obtenido por otros Tuits de la misma cuenta sobre aspectos de didáctica de las ciencias que no mencionaban artículos de didáctica.

\section{Resultados}

\section{Valoración de las publicaciones de didáctica por parte del profesorado}

La inclusión de publicaciones tuvo un impacto distinto en cada una de las modalidades, siento muy valoradas en general en aquéllas modalidades que incluyeron espacios de discusión colectiva, la Discusión en marco formativo y el Acompañamiento en marco formativo.

En lo que respecta a las otras modalidades de inclusión, poner simplemente revistas a disposición del profesorado (modalidad Disponibilidad) pareció tener poco efecto. Se observó mediante marcadores de papel el movimiento de los ejemplares, y se detectaron sólo 9 consultas en todo el curso académico. Tanto en la Invitación en marco formativo como el Acompañamiento en marco formativo, los participantes mostraron mucho interés e incluso sorpresa, ya que algunos de ellos afirmaban no tener conocimiento de este tipo de recurso. Los comentarios del profesorado en las distintas actividades formativas colectados en las encuestas evidencian la reflexión y conexión sobre la labor docente que desencadenó la lectura de artículos, abundando expresiones relativas al trabajo conjunto del profesorado:

"Desconocía este recurso, y me ha servido mucho para buscar y encontrar no sólo recursos, sino también metodologías. Lo cierto es que he aplicado bastantes metodologías que he leído y me han cambiado la forma de hacer las clases. Creo que es un recurso que usamos poco (el profesorado en general) y eso no nos ayuda a trabajar conjuntamente buscando otras metodologías y compartir recursos".

"Eso que describen en este artículo yo lo he hecho en clase, no me salió bien y pensé que habíamos perdido el tiempo. [...]Ahora veo que aunque no te salga, puedes hablar con los alumnos de por qué sale una cosa u otra y aprenden más".

"Pienso que la lectura y reflexión de los artículos sobre didáctica nos hacen darnos cuenta de los marcos teórico-prácticos en que nos movemos, nos obligan inconscientemente a posicionarnos y reflexionar sobre la práctica docente. Pienso que eso tiene sentido si se comparte con los otros y se contrastan puntos de vista. [...]. Me he sentido muy bien en la actividad [...]".

"La calidad del artículo y su aplicabilidad al aula varían mucho entre los distintos casos. La dificultad principal es que, aun cuando el artículo es interesante, hay que hacer un sobreesfuerzo para aplicarlo realmente en el aula. Es fácil quedarse con el "mira qué interesante" y seguir con lo de siempre. Trabajar en grupo ayuda. Tienes que terminar aplicándolo porque has quedado con otro y lo harás y discutirás".

En las 83 encuestas recogidas entre el profesorado participante, los resultados conjuntos indican (Figura 2) que en general el profesorado considera que los artículos de didáctica tratan temáticas relacionadas directamente con la docencia (con un apoyo medio de 5) y son de utilidad $(4,6)$. Por lo que respecta a los artículos como vía de profesionalización, el profesorado considera que leer artículos de didáctica ayuda a valorar más $(4,3)$ y prestar más atención $(4,8)$ a la propia actividad docente. Al comparar los datos obtenidos en las distintas formaciones, observamos que de forma general las valoraciones de los profesores participantes en las modalidades que incluyen espacios de discusión (Acompañamiento o Discusión) son las que obtienen en los distintos ítems una 
puntuación más alta, mientras que las modalidades de Invitación o Disponibilidad resultan en valoraciones más modestas.

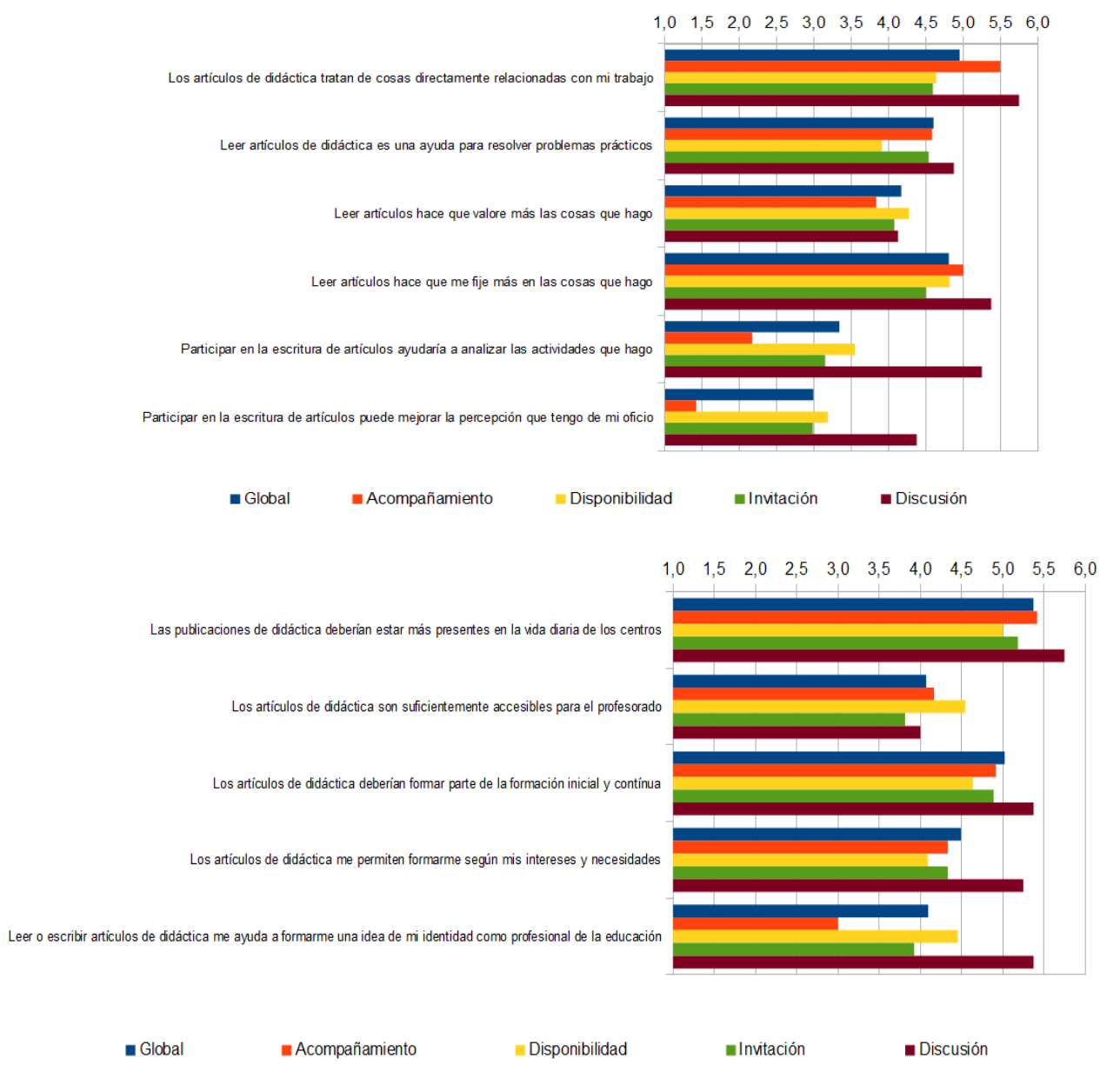

Figura 1.- Medias de valoración de los distintos ítems en la encuesta según la actividad de formación. Cada valoración se representa identificada con un color distinto según se indica en la leyenda. Se ofrecen también los valores medios ponderados como "Global".

De forma general, independientemente de la modalidad, el profesorado participante considera que los artículos de didáctica deberían estar más presentes en la vida de los centros educativos $(5,4)$ y las actividades de formación (5), si bien asume que las publicaciones de didáctica son accesibles para el profesorado $(4,1)$ y permiten formarse según los propios intereses $(4,5)$, lo que sugiere que su ausencia de los entornos de práctica y formación no se debe a dificultades en el acceso. Los ítems en que se valora la utilidad de participar en la escritura de artículos de didáctica como herramienta de análisis de la propia práctica, mejorar la percepción del oficio y forjar una identidad como profesional de la educación son los que obtienen una valoración más baja (3,2. 3,4 y 4,1, respectivamente), y en los que la diferencia entre la modalidad de Discusión y las demás modalidades de inclusión de artículos en la formación se hace más evidente.

De forma general, las encuestas recogidas muestran que el profesorado participante estaba formado principalmente $(78 \%)$ por profesores que antes de la formación leían artículos de didáctica de forma ocasional (1-4 artículos por curso) y pocos que los leían nunca (3\%); o a menudo (más de 4 artículos por curso) (19\%). Aunque no se preguntó en la encuesta específicamente por el marco en el 
que se realizaban estas lecturas, en conversaciones con el profesorado se identificó como origen de estas lecturas la búsqueda de actividades y recursos educativos mediante internet, $\mathrm{y}$, en menor medida, cursos de formación.

\section{Análisis de las elecciones de artículos de didáctica por parte de los profesores}

La Discusión en marco formativo llevada a cabo en el grupo de trabajo EduWikilab tuvo muy buenos resultados. Las breves presentaciones de artículos no sólo resultaron de interés para los participantes, sino que identificaron puntos de interés previos y vías de acción futura. A lo largo de las sesiones, los participantes propusieron artículos de nuevas revistas publicadas en abierto no previstas inicialmente, las revistas PLOS ONE. Science Education y el European Journal of Physics, que fueron incorporadas a la librería digital.

De los 42 artículos elegidos libremente por el profesorado por su interés, la mitad describían propuestas didácticas de aplicación en el aula y la otra mitad se referían a marcos teóricos o pedagógicos (Tabla 2). Las categorías temáticas se vieron influenciadas por las líneas de trabajo del grupo -en ese momento centrado en el trabajo con vídeos en las ciencias y el diseño de actividades de lectura en ciencias- de modo que se identifican dos ejes en las elecciones de artículos por parte del profesorado: el tipo de actividades que está intentando desarrollar en este momento, y dos ejes metodológicos principales en la enseñanza de las ciencias, esto es, las actividades manipulativas y la enseñanza mediante la indagación.

Tabla 2.

Clasificación por categorías de los artículos elegidos por el profesorado.

\begin{tabular}{lccccccc}
\hline & $\begin{array}{c}\text { TIC y } \\
\text { Multimedia }\end{array}$ & $\begin{array}{c}\text { Lectura, } \\
\text { escritura y } \\
\text { oralidad en } \\
\text { ciencias }\end{array}$ & $\begin{array}{c}\text { Comp. } \\
\text { Básicas y } \\
\text { PISA }\end{array}$ & Evaluación & $\begin{array}{c}\text { Laboratorio y } \\
\text { actividades } \\
\text { manipulativas }\end{array}$ & Indagación & $\Sigma$ \\
\hline $\begin{array}{c}\text { Propuestas } \\
\text { didácticas } \\
\begin{array}{c}\text { Marcos } \\
\text { teóricos o } \\
\text { pedagógicos }\end{array}\end{array}$ & 4 & 2 & - & - & 10 & 5 & 21 \\
\hline
\end{tabular}

Nota: los artículos han sido seleccionados libremente por el profesorado en el marco de la actividad del grupo EduWikiLab por su interés en categorías temáticas (TIC, Lectura, CCBB,...) y tipo (Propuestas didácticas o marcos teóricos).

Se detecta también que las revistas académicas (Revista Electrónica de Enseñanza de las Ciencias, Enseñanza de las Ciencias) son usadas menos veces (1 ó 2 veces, respectivamente) que las técnico-profesionales, cada una de las cuales es usada varias veces, 4 (Alambique, Science in School, Recursos de Física), 6 (Revista Ciències) o hasta 8 veces (Revista Eureka).

\section{Análisis del contenido de revistas de didáctica específica de las ciencias}

Las revistas de didáctica específica analizadas muestran grandes diferencias entre sí, de modo que las de perfil técnico-profesional inciden mucho en propuestas de actividades didácticas (Revista Ciències, Eureka, Alambique), mientras que las de perfil académico se centran más en la detección de concepciones del alumnado y profesorado y estudio de marcos y líneas pedagógicas (Tabla 3). En estas últimas, los artículos sobre análisis de libros de texto y concepciones de alumnado de magisterio están altamente representados (entre un $21 \%$ y un $34 \%$ del total, dependiendo de la revista), cosa se debe seguramente al acceso logísticamente sencillo para los investigadores, ubicados en universidades. 
Tabla 3.

Distribución por categorías temáticas de los artículos de distintas revistas del ámbito académico-profesional de la enseñanza de las ciencias.

\begin{tabular}{|c|c|c|c|c|c|c|c|}
\hline & $\begin{array}{c}\text { Concepciones } \\
\text { y análisis de } \\
\text { alumnado de } \\
\text { prim. } / \text { sec. }\end{array}$ & $\begin{array}{l}\text { Análisis } \\
\text { de libros } \\
\text { de texto }\end{array}$ & Currículum & $\begin{array}{l}\text { Concepciones } \\
\text { y análisis de } \\
\text { alumnado de } \\
\text { magisterio }\end{array}$ & $\begin{array}{l}\text { Propuestas } \\
\text { didácticas } \\
\text { de aula }\end{array}$ & $\begin{array}{c}\text { Marcos } \\
\text { teóricos o } \\
\text { pedagógicos }\end{array}$ & $\begin{array}{l}\text { Concepciones } \\
\text { y análisis de } \\
\text { profesorado } \\
\text { de prim./sec. }\end{array}$ \\
\hline $\begin{array}{l}\text { Enseñanza de } \\
\text { las Ciencias }\end{array}$ & 14.9 & 8.5 & 4.3 & 17.0 & 19.1 & 36.2 & 0.0 \\
\hline $\begin{array}{c}\text { Enseñanza de } \\
\text { las Ciencias de } \\
\text { la Tierra }\end{array}$ & 0.0 & 0.0 & 5.7 & 0.0 & 25.7 & 68.6 & 0.0 \\
\hline Revista Ciències & 0.0 & 2.9 & 0.0 & 0.0 & 65.7 & 31.4 & 0.0 \\
\hline $\begin{array}{c}\text { Revista } \\
\text { Electrónica de } \\
\text { Enseñanza de } \\
\text { las Ciencias }\end{array}$ & 21.9 & 9.4 & 0.0 & 25.0 & 18.8 & 15.6 & 9.4 \\
\hline $\begin{array}{c}\text { Revista Eureka } \\
\text { de Enseñanza y } \\
\text { Divulgación de } \\
\text { las Ciencias. }\end{array}$ & 8.9 & 15.6 & 0.0 & 13.3 & 33.3 & 28.9 & 0.0 \\
\hline $\begin{array}{c}\text { Alambique. } \\
\text { Didáctica e las } \\
\text { Ciencias } \\
\text { Experimentales }\end{array}$ & 3.3 & 0.0 & 3.3 & 3.3 & 63.3 & 23.3 & 3.3 \\
\hline $\begin{array}{l}\text { Recursos de } \\
\text { Física }\end{array}$ & 0.0 & 0.0 & 0.0 & 0.0 & 85.7 & 14.3 & 0.0 \\
\hline $\begin{array}{c}\text { Science in } \\
\text { School }\end{array}$ & 2.4 & 0.0 & 2.4 & 0.0 & 46.3 & 46.3 & 2.4 \\
\hline
\end{tabular}

Nota: Han sido analizados entre 30 y 45 artículos por revista, se presenta el porcentaje de artículos de cada categoría temática en cada una de las revistas.

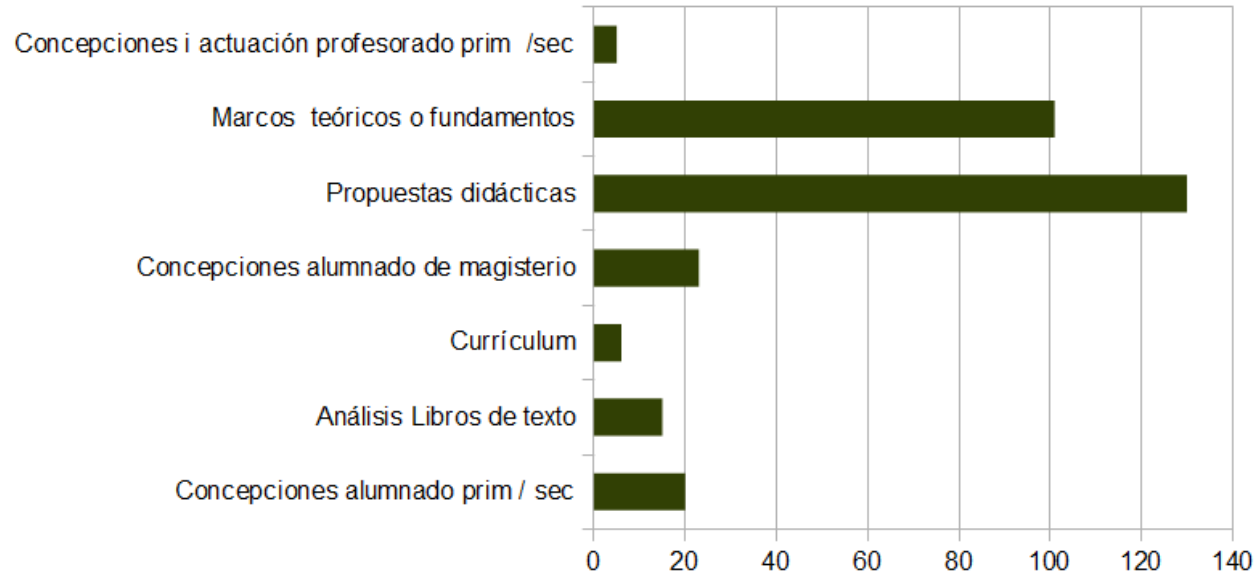

Figura 2. Distribución temática del total de 300 artículos de las distintas revistas. El gráfico representa los valores absolutos: el total de artículos identificado en cada categoría.

De manera global (Figura 2), más de la tercera parte de los artículos se dedica a la propuesta y análisis de actividades didácticas de aplicación en el aula, y una tercera parte a la descripción o discusión de marcos teóricos o líneas pedagógicas, temáticas ambas de interés formativo inmediato o a 
largo plazo para el profesorado, aunque como hemos visto, la distribución de estas categorías entre revistas es desigual.

Debe tenerse en cuenta que en la categoría de marcos teóricos o fundamentos, en las revista Enseñanza de las Ciencias de la Tierra y Science in School, a diferencia de las demás, la mayoría de artículos no se refieren a marcos pedagógicos, sino disciplinares, de actualización científica.

\section{Inclusión en entorno TIC y análisis de cibermetría}

El número de visitas recibidas por los post con recopilaciones de artículos de didáctica se mantuvo alrededor de la media de visitas obtenidas en el mismo período por el resto de post de temáticas distintas (descripción de materiales educativos, plantillas, actividades y reflexiones pedagógicas) del mismo blog. En cambio, se detectaron variaciones importantes en función de la temática del recopilatorio (Figura 3).

El conjunto de visitas a los postrecopilatorios suma 230 visitas. En cambio, los enlaces contenidos en los post que conducen a los artículos en abierto han acumulado sólo 83 accesos, lo que indica que de las 230 visitas, al menos 150 no llegaron realmente a consultar ningún artículo (un 64\%), y el resto de visitas realizaron una media de una sola consulta por lectura del post. En la difusión de los post en Twitter mediante la cuenta del autor@jdomenechca, los Tweets referentes a lecturas sobre didáctica específica obtuvieron un impacto (medido en \%Engagement ${ }^{4}$ ) de 4,1 , por encima pero muy similar a la media del resto de Tweets de la cuenta $(3,8 \%)$, referidos a reflexiones pedagógicas y recursos didácticos en el mismo período.

\section{Discusión y conclusiones}

El profesorado valora positivamente los artículos de didáctica como recurso formativo y considera que deberían estar más presentes en la vida de los centros y actividades formativas. A diferencia de los tópicos generales según los cuales el profesorado está principalmente interesado en los aspectos prácticos, nuestros resultados muestran que, pudiendo elegir libremente, el profesorado da la misma importancia a los artículos que presentan experiencias didácticas que a los que describen o discuten marcos teóricos. Así mismo, el profesorado valora positivamente la accesibilidad de los artículos de didáctica.

A pesar de eso, tanto la encuesta como el estudio de cibermetría detectan que, aun siendo consciente de su utilidad y accesibilidad, el profesorado no recurre a la consulta de artículos de didáctica de forma habitual. Incluso en entornos TIC, donde los artículos de didáctica reciben aparentemente una atención similar a otros recursos didácticos, no llegan realmente a ser consultados por el profesorado.

Los resultados muestran que la modalidad de uso elegida en la formación tiene efectos importantes en las visiones del profesorado en relación a los artículos de didáctica, en especial en lo referente a la adquisición de un rol profesional. En particular, los profesores tienen mejor percepción de los artículos de didáctica cuando se proporciona un espacio de lectura colectiva, orientada a la discusión en grupo, bien en torno a temáticas concretas y compartidas (los casos de las actividades de formación IndComp y Ventanas Moodle) o bien cuando se ciñe a los propios intereses del profesorado 
(el caso EduWikiLab). En cambio, su simple propuesta abierta sin la creación de espacios de discusión o la difusión mediante las TIC conllevan una valoración más baja o un menor acceso real a los artículos.

Con respecto al contenido de las revistas de didáctica (en este caso, de didáctica específica de las ciencias) una parte importante (más de dos tercios) de los artículos analizados se ocupan de temáticas susceptibles de ser relevantes para el profesorado (marcos pedagógicos y actividades didácticas), si bien hay grandes diferencias según el tipo de revista. El profesorado elige preferiblemente artículos de revistas de tipo técnico-profesional, independientemente de que elija artículos de descripción de actividades (más frecuentes en estas revistas) o artículos de marcos teóricos o pedagógicos (más frecuentes en las académicas). Posiblemente se deba a que en las revistas técnicoprofesionales como Ciències, Cuadernos de Pedagogía o Aula de secundaria el formato del texto es narrativo y experiencial, mientras que en las revistas académicas es habitual el formato OMRC (objetivos, metodología, resultados y conclusiones), mucho más estructurado y de lectura un tanto más farragosa. Como se ha dicho anteriormente, la riqueza (y requerimiento) de referentes pedagógicos en estas últimas revistas puede también estar actuando de freno en su lectura por parte de los profesores.

\section{Implicaciones}

Lo descrito en el apartado anterior evidencia un alejamiento de la valoración teórica de las revistas por parte del profesorado y su uso en la práctica, en la que las redes sociales y la formación horizontal toman el relevo, con el riesgo de establecer múltiples núcleos de innovación e investigación educativa desconectados entre sí y de las líneas y marcos pedagógicos. Consideramos que son necesarias acciones para conectar la praxis con la teoría mediante las revistas de didáctica y esbozamos partiendo de nuestra experiencia en centros docentes, algunas líneas de actuación más allá de la inclusión de las revistas en actividades de formación:

- Los espacios y reuniones del profesorado en los centros de secundaria (Equipos docentes, Departamentos) pueden articularse como espacios de aprendizaje conjunto con pequeñas intervenciones (Antúnez et al, 2002; López, Sánchez y Altopiedi, 2011, Domènech-Casal, 2013). Introducir en ellos la lectura y discusión de artículos de didáctica dirigida a problemas concretos es una vía de profesionalización y creación de una mirada pedagógica a la propia práctica.

- La elaboración por parte de las propias revistas de "packs temáticos" de artículos en abierto agrupados por etiquetas (por ejemplo, modelización, evaluación,...) para su uso en formaciones del profesorado o "reviews" temáticos dirigidos al profesorado alrededor de un aspecto disciplinar concreto (la estructura del átomo, la fórmula de la velocidad,...) en sus varios aspectos (concepciones del alumnado, actividades didácticas, marcos teóricos...) facilitaría su inclusión en las actividades de formación.

- Mejorar el reconocimiento a la publicación de artículos y establecer vías de apoyo al profesorado (por ejemplo, mediante la ayuda de técnicos de los CRP que ayudaran a formalizar el discurso) para que comunique sus innovaciones didácticas son vías que deberían estudiarse, en particular cuando existen en la bibliografía experiencias exitosas de investigación-acción en este sentido (Imbernón, 2002; Solbes y Souto, 1999). Establecer vías, mediante CRP o inspección, para conocer qué publicaciones genera el cuerpo de profesorado podría suponer una eficaz y poco costosa herramienta para saber qué conocimiento que se genera en el sí de la institución.

Los resultados de este artículo se extraen de los resultados de un grupo pequeño (83 profesores) y heterogéneo de profesorado y del análisis de experiencias circunscritas a ámbitos temporales (cibermetría) o didácticas específicas (revistas de didáctica de las ciencias). No ha sido posible hacer análisis en relación a otros factores (edad, género, años de ejercicio,...) porque no se dispone de esos datos. Esto hace que las conclusiones no sean necesariamente extrapolables a otros grupos de profesores o ámbitos disciplinarios. Así mismo, la naturaleza breve de la experiencia y la falta de medios para hacer un seguimiento del profesorado participante hacen que no podamos ofrecer 
una medida objetiva del impacto que ha tenido la lectura de los artículos de didáctica en la praxis real del profesorado participante. Consideramos que el uso de datos de cibermetría usado de forma tentativa en este artículo puede proponerse como metodología de estudio para arrojar más luz sobre el uso real por parte del profesorado de artículos de didáctica.

En todo caso, los resultados muestran que la valoración de la inclusión de los artículos de didáctica en actividades formativas depende en gran medida de la modalidad en que se incluyan. Estimamos evidente que los artículos de didáctica y su discusión deberían ser un elemento fundamental en cualquier actividad formativa inicial o continua del profesorado, y que es necesaria una formación específica sobre qué revistas existen y cómo usarlas para la creación de conocimiento pedagógico, en consonancia con la función emancipadora de la formación y el rol de creador de conocimiento pedagógico que necesitamos del profesorado.

\section{Agradecimientos}

El autor agradece a los profesores participantes a las actividades de formación continua EduWikiLab, IndComp, Ventanas Moodle y Experimentació a les Aules y al profesorado del INS Vilanova del Vallès su participación y colaboración. Se agradece a la la revista Cuadernos de Pedagogía su colaboración en la experiencia. Este artículo se enmarca en la actividad del autor en el grupo LIEC (Llenguatge i Ensenyament de les Ciències), grupo de investigación consolidado LICEC (referencia 2014SGR1492) de la Universitat Autònoma de Barcelona porAGAUR (Agència d'Ajuts Universitaris i de Recerca) y financiado por el Ministerio de Economía y Competitividad (referencia EDU2015-66643-C2-1-P).

\section{Referencias}

Andreis, A.M. (2013). La constitución del profesor: el escribir como investigación sobre su actuación. Investigación en la escuela, 81, 79-89.

Antúnez, S., Bonals, J., CEIP Seat Infantil, Cerdà, R., Fernánez, F. J., Mauri, T., y Tirado, V. (2002). Dinámicas colaborativas en el trabajo del profesorado. El paso del yo al nosotros. Barcelona, España: Graó.

Armengou, M. E., Castillo, J., Costa, M. M., Donat, D., Martí, L., Mediavilla, U. J., Pano, O., Sanz, J., Vázquez, M., Casas, A., y Domènech-Casal, J. (2015). "Lo que no sabemos" y "lo que ya hacemos": una exploración intercentros para aprender a evaluar y enseñar por competencias. Cuadernos de Pedagogía, 461, 34-39.

Barba-Martín, R. A., Barba, J. J., Martínez, S. (2016). La formación contínua colaborativa a través de la investigación-acción. Una forma de cambiar las prácticas de aula. Contextos educativos, 19, 161-175.

Carrascosa-Alís, J., Martínez-Torregrossa, J., Furió-Mas, C. y Guisasola, J. (2008). ¿Qué hacer en la formación inicial del profesorado de ciencias en secundaria? Revista Eureka sobre Enseñanza y Divulgación de las Ciencias, 5(2), 118-13.

Carro, L. (2000). La formación del profesorado en investigación educativa: una visión crítica. Revista Interuniversitaria de Formación del Profesorado, 68(24,2), 103-122.

DOGC (2014). Resolució ENS/225/2014 de convocatòria de concurs de trasllats adreçada al personal funcionari de carrera. Diari Oficial de la Generalitat de Catalunya, 6739, 1-98.

Domènech-Casal, J. (2013). Innovación y formación en un mundo en crisis: ¿Qué podemos hacer en y desde los centros educativos? Organización y Gestión Educativa, 3, 6-11.

Domènech-Casal, J. (2016). Las redes sociales como forja del rol profesional del docente. Cuadernos de Pedagogía, 469, 31-34.

European Comission (2015). The Teaching Profession in Europe: Practices, Perceptions, and Policies. Eurydice Publications.

[https://webgate.ec.europa.eu/fpfis/mwikis/eurydice/index.php/Publications:The Teaching_Profession in Europe:_Practices,_Perceptions,_and_Policies ]

Forner, A. (2000) Investigación educativa y formación del profesorado. Revista Interuniversitaria de Formación del Profesorado, 39, 33-50. 
Furió, C. y Gil, D. (1998). Hacia la formulación de programas eficaces en la formación continuada del profesorado de Ciencias. Educación Científica (Actas del Congreso Iberoamericano de Educación en Ciencias Experimentales. Formación Permanente de Profesores) 129-146. Alcalá, España: Servicio de publicaciones de la Universidad de Alcalá.

Greenwood, D.J. (200). De la observación a la investigación-acción participativa: una visión crítica de las prácticas antropológicas. Revista de Antropología Social, 9, 27-49.

Guisasola, J., Pintos, M. E., y Santos, T. (2001). Formación continua del profesorado, investigación educativa e innovación en la enseñanza de las ciencias. Revista Interuniversitaria de Formación del Profesorado, 41, 207-222.

Hargreaves, A., y Shirley, D. (2012). La cuarta vía. El futuro prometedor del cambio educativo. Barcelona: Octaedro.

Imbernón, F. (Coord). (2002). La investigación educativa como herramienta de formación del profesorado. Reflexión y experiencias de investigación educativa. Barcelona, España: Graó.

INEE (2014). TALIS 2013: Estudio internacional sobre la Enseñanza y el Aprendizaje - Informe internacional. Boletín de Educación EducaINEE, 34.

Innerarity, D. (2011). La democracia del conocimiento. Por una sociedad inteligente. Barcelona, España: Espasa Libros.

Lavié, J. M. (2012). Organizar los centros para el aprendizaje del profesorado: cinco reflexiones. Organización y Gestión Educativa, 20(3), 14-18.

López, J., Sánchez, M., y Altopiedi, M. (2011). Comunidades profesionales de práctica que logran sostener procesos de mejora institucional en las escuelas. Revista de Educación, 365, 109-131.

Marina, J. A., Pellicer, C., y Manso, J. (2015). Libro Blanco de la Profesión Docente y su entorno escolar. En: [http://blog.educalab.es/intef/wp-content/uploads/sites/4/2015/12/LIBROBLANCO ProfesionDocente JAM v11.pdf]

Moon, J. A. (2006). Learning journals. A handbook for reflective practice and professional development. Nueva York, Routledge Falmer.

OECD (2013). New Insights from TALIS 2013: Teaching and Learning in Primary and Upper Secondary Education. OECD Publishing. [http://dx.doi.org/10.1787/9789264226319-en]

OECD (2015). Embedding profesional development in schools for teacher success. Teaching in focus, TALIS, 10.

Oliva, J. M. (2011). Dificultades para la implicación del profesorado de Educación Secundaria en la lectura, innovación e investigación en didáctica de las ciencias (I): el problema de la inmersión. Revista Eureka sobre Enseñanza y Divulgación de las Ciencias, 8(1), 41-53.

Oliva, J. M. (2012). Dificultades para la implicación del profesorado de Secundaria en la lectura, innovación e investigación en didáctica de las ciencias (II): el problema del "manos a la obra". Revista Eureka sobre Enseñanza y Divulgación de las Ciencias, 9(2), 241-251.

Palos, J., Martínez, M., y Albadalejo, C. (2012). Las nuevas tendencias de la formación permanente del profesorado. Aula de Innovación Educativa, 212, 27-32.

Perrenoud, P. (2010). La formación del profesorado: un compromiso entre visiones inconciliables de la coherencia. Revista Interuniversitaria de Formación del Profesorado, 68 (24,2) 103-122.

Rodríguez-Gómez, D. (2011). La gestión del conocimiento en las organizaciones educativas. Revista Catalana de Pedagogia, 7, 435-448.

Sancho, J. M. y Alonso, C. (Eds.) (2012). La fugacidad de las políticas, la inercia de las prácticas. La educación y las tecnologías de la información y la comunicación. Barcelona, España: Octaedro.

Sanmartí, N. (2008). Contribuciones y desafíos de las publicaciones del área de educación en ciencias en la construcción y consolidación de la identidad del área: la experiencia de la revista Enseñanza de las Ciencias. Enseñanza de las Ciencias, 26(3), 301-310.

Sein-Echaluce, M. L., García, F. J., y Fidalgo, A. (2014). Indicadores y Características de la Innovación Educativa. Encuesta MOOC de "Innovación Educativa Aplicada. En: [https://innovacioneducativa.files.wordpress.com/2015/05/encuesta-de-indicadores-ycaracterc3adsticas-de-la-innovacic3b3n-educativa-v2.pdf] 
Solbes, J. y Souto, X. M. (1999). Investigación desde la escuela y formación del profesorado. Investigación en la Escuela, 38, 87-98.

Solbes, J., Domínguez-Sales, M. C., Fernández-Sánchez, J, Furió, C., Cantó, J. R., y Guisasola, J. (2013) ¿El profesorado de física y química incorpora los resultados de la investigación en didáctica? Didáctica de las ciencias experimentales y sociales, 27, 155-178.

Tejada, J. (2009). Competencias docentes. Profesorado. Revista de currículum y formación del profesorado, 13(2), 1-15.

Zeichner, K. M. (1993). El docente como profesional reflexivo. Cuadernos de Pedagogía, 220, 44-49.

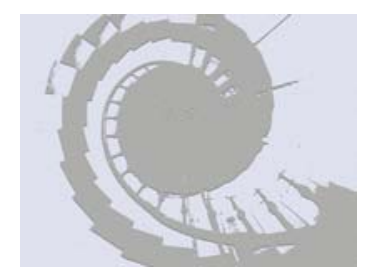

1 Grupo de Trabajo de Profesorado de Ciencias EduWikiLab https://eduwikilab.wordpress.com/

2 Seminarios de Lectura de Didáctica EduWikiLab

https://eduwikilab.wordpress.com/category/seminaris-de-didactica/

$3 \quad$ Biblioteca de Revistas de Didáctica de las Ciencias

https://eduwikilab.wordpress.com/2013/07/20/biblioteca-de-revistes-de-didactica-de-les-ciencies/

$4 \quad$ El término Engagement identifica la situación en la que un usuario realiza alguna acción (clicar sobre el nombre el autor, clicar el enlace que contiene, retuitear, clicar sobre algún hashtag...) sobre un Tuit publicado por otro usuario. El número de usuarios que realizan algún Engagement sobre un Tuit, en relación al número de usuarios que lo han visto (\%Engagement) se utiliza de forma habitual como medida aproximativa del impacto de un Tuit. 